\title{
High-resolution radio observations of nearby galaxies
}

\author{
D.J. Saikia \\ Tata Institute of Fundamental Research, NCRA, Post Bag 3, Ganeshkhind, Pune 411 007, India \\ and
}

A. Pedlar

Nuffield Radio Astronomy Laboratories, Jodrell Bank, Macclesfield, Cheshire SK11 9DL, UK

The central regions of many nearby active galaxies are often heavily obscured by dust and gas and are visible at only the far infrared (FIR) and radio wavelengths. High-resolution radio observations provide an invaluable tool for clarifying the dominant power source in an active galaxy which could be due to either an intense burst of star formation or an active galactic nucleus (AGN). An AGN is normally assumed to be powered by a supermassive black hole with an accretion disk, where the black hole forms as the end product of stellar evolution. There have been suggestions linking both these forms of activity to galaxy interactions, but the conditions that might lead to the formation of an AGN and how it is fuelled are not well understood.

The observed FIR emission in these galaxies is largely due to dust which absorbs the radiation from the population of hot young stars and re-radiates it at infrared wavelengths. On the other hand, the extended sources of non-thermal radio emission are due to acceleration of particles by supernovae and the subsequent evolution of the supernova remnants (SNRs), while the compact radio sources could be due to young SNRs, compact HII regions or AGN. Several diagnostic tests are however required to clarify the nature of these compact radio sources. The brightness temperature of a compact source produced by a starburst is less than about $10^{5} \mathrm{~K}$ and its radio spectrum becomes flat when the brightness temperature significantly exceeds the electron temperature of about $10^{4} \mathrm{~K}$. On the other hand, non-thermal sources associated with an AGN have flat radio spectra when synchrotron self-absorption becomes important at brightness temperatures exceeding about $10^{10} \mathrm{~K}$. The radio structures can also provide a useful diagnostic to identify whether there is an AGN in the nuclear region of a galaxy. For example, well-collimated radio jets such as the ones seen in Mkn3 (Kukula et al 1993) which traverse outwards from a flat-spectrum nuclear component (Ghosh et al., in preparation) must be due to an AGN since starbursts cannot produce such structures. The radio brightness distribution of a starburst galaxy is intimately related to the distribution of massive stars, but could be broadened by the diffusion of cosmic-ray particles due to supernova-driven winds as well as the expansion and evolution of old remnants. Another useful diagnostic for identifying whether an AGN is the dominant source of power is to look for significant departures from the very tight FIR-radio luminosity correlation which spans several decades of luminosity (see

T. J.-L. Courvoisier and A. Blecha: Multi-Wavelength Continuum Emission of AGN, 189-192.

(C) 1994 IAU. Printed in the Netherlands. 


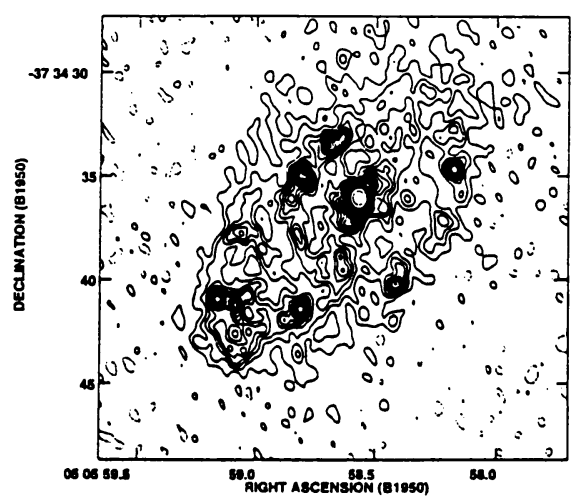

Fig. 1. Radio image of the starburst galaxy NGC1808 at $8.4 \mathrm{GHz}$

Condon 1992 for a review).

Radio images of two of the archetypal starburst galaxies NGC253 and NGC1808 observed with the VLA at $8.4 \mathrm{GHz}$ (Collison et al., in preparation), show the compact radio components in the nuclear region. Comparison of these images with earlier observations of NGC253 (Antonucci \& Ulvestad 1988) and NGC1808 (Saikia et al. 1990) at $5 \mathrm{GHz}$ suggests that most of the components have spectral indices $\alpha \geq 0.4\left(\mathrm{~S} \propto \nu^{-\alpha}\right)$, suggesting that they are SNRs occurring in regions of high star formation. In order to identify a larger sample of such galaxies and also those which might be harbouring an AGN, and understand the conditions which could lead to the formation of an AGN, we started a project to study the properties of a sample of galaxies with morphologically peculiar nuclei (Saikia et al., in preparation). These galaxies, which have been christened Sérsic-Pastoriza or S-P galaxies include galaxies with diffuse and amorphous nuclei in addition to the well-known hot-spot systems. They reflect a broad spectrum of properties with some of them also having a Seyfert nucleus. A radio survey of a sample of about 50 such galaxies with an angular resolution of about an arcsec shows that while almost all the ones with prominent hot-spots are detected as radio sources, those with weak nuclei and no well-defined hot-spots at optical wavelengths are rarely detected. Compact radio components have been seen in most of the galaxies detected as radio sources. Using the peak brightness of the components and assuming their sizes to be similar to the resolution of the observations, the brightness temperatures are invariably less than about $10^{5} \mathrm{~K}$, and hence cannot be identified unambiguously with AGN. The steep radio spectra of the individual peaks of emission for which we could determine spectral indices suggest that most of them are likely to be SNRs. The distributions of the luminosities of the compact components observed with the highest resolution at, say, $8 \mathrm{GHz}$, show that they are somewhat more luminous than those in M82 (Muxlow et al. 1993), where the median value is about $10^{18} \mathrm{~W} \mathrm{~Hz}^{-1}$. This is significantly higher than for SNRs in the LMC and in our Galaxy by about an order of magnitude. For the more distant galaxies, only the most luminous ones 


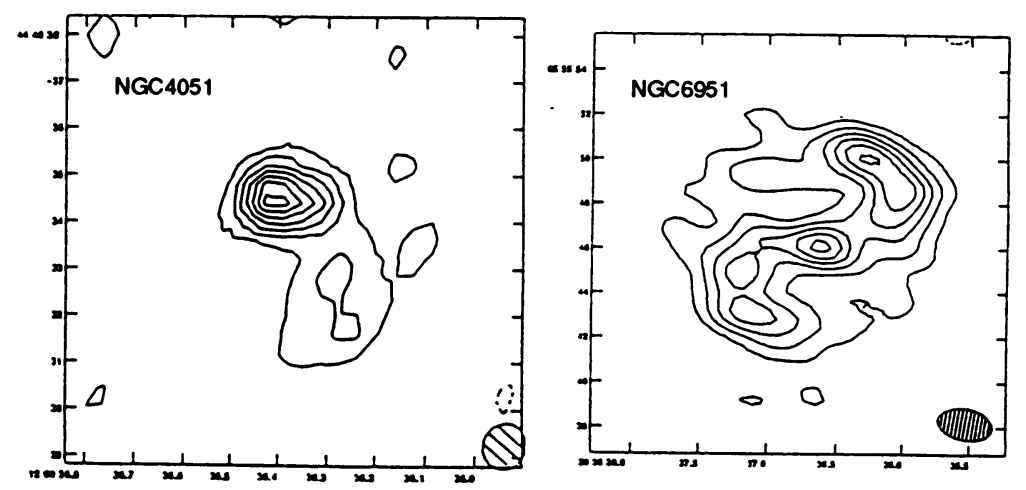

Fig. 2. Radio images of the Sérsic-Pastoriza galaxies NGC4051 and NGC6951

are detected with the weaker ones being either below the detection limit or confused by the extended emission. The luminosites of these putative SNRs are, however, smaller than the compact components in the nuclear regions of Seyfert galaxies (Kukula et al., in preparation). Of the four most luminous components seen in our sample of S-P galaxies, NGC2196 has a compact flat-spectrum component with $\alpha \sim 0.1$, NGC613 has a weak nuclear component (Hummel \& Jörsäter 1992) while NGC2782 and NGC3504 have dominant compact components surrounded by more extended diffuse emission. We also find evidence of weak radio rings in the galaxies NGC1530, NGC2997 and NGC3351, in addition to the well-known ones in NGC613 (Hummel \& Jörsäter 1992) and NGC1365 (Sandqvist et al., in preparation). The formation of rings could be due to a bar which causes these structures to form at the Inner Lindblad Resonance. The radio continuum emission appears to trace a spiral pattern in the nuclear regions of NGC5430 and NGC6951.

In the nearby galaxy M82, MERLIN observations have revealed details of the structure of individual SNRs. For example, at $5 \mathrm{GHz}$ a majority of the 23 detected SNRs show evidence of shell or partial shell structures. Their surface brightnessdiameter relation is consistent with that seen for Galactic and LMC SNRs with the ones in M82 extending the relation to smaller diameters and higher values of surface brightness. The plot of the cumulative numbers of SNRs less than the diameter $\mathrm{D}$ is linear for the 23 detected remnants whose sizes range from about 0.5 to $4.5 \mathrm{pc}$ suggesting that they are in a state of free expansion. The flux density of the SNRs is inversely proportional to its diameter, which is inconsistent with simple adiabatic losses in a synchroton emitting source (Muxlow et al. 1993).

High-resolution radio observations at different frequencies have played an important role in identifying possible AGN and establishing the close alignment of the radio structures and the cones of emission seen at optical wavelenghts (e.g. Pedlar et al. 1993 for NGC4151; Hummel \& Saikia 1991 for NGC4388). The orientation of the cones relative to the plane of the galaxy, however, varies over a wide range being nearly orthogonal in NGC 4388 to being closer to the plane in 

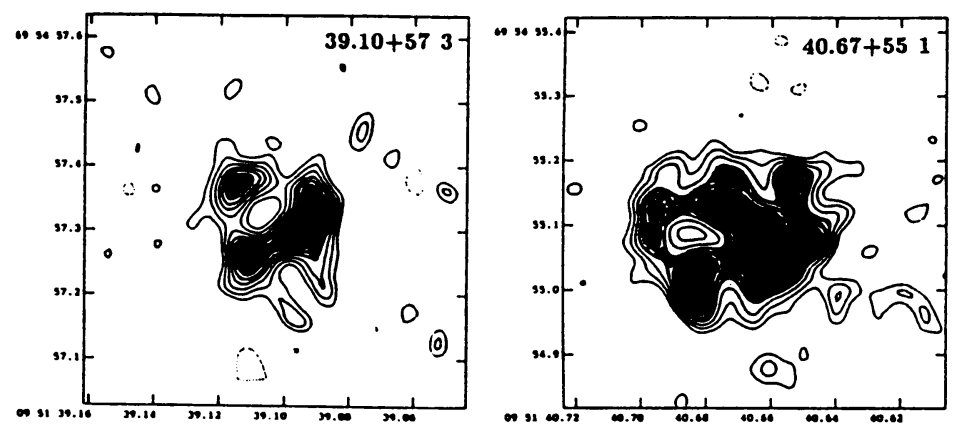

Fig. 3. MERLIN images at $5 \mathrm{GHz}$ of two of the remnants in M82

\section{NGC4151.}

In the somewhat more distant low-luminosity Fanaroff-Riley class I radio galaxies the radio axes tend to be along the optical minor axes of the parent elliptical galaxies. However, this minor axis trend appears to be almost entirely due to sources with prominent cores whose contribution to the total flux density at, say $2.7 \mathrm{GHz}$, is greater than about 10 per cent (Kapahi \& Saikia 1982). For those with weaker cores, there does not seem to be a significant trend. The tendency for sources with stronger cores to be aligned with the minor axes may be related to the efficiency of fuelling in the nucleus depending on the relative orientation of the accretion disk, stellar and gas rotation axes and the parent galaxy. Highresolution radio observations with the VLA $A$-array at $\lambda 20,6,2$ and $1.3 \mathrm{~cm}$ of about 30 galaxies selected from the earlier sample with roughly equal number of sources with weak and strong cores confirm the earlier result and show no significant difference in the spectra of the cores (Saikia et al., in preparation). In these high-resolution observations, only the bases of the jets are often detected and they are usually very asymmetric if not completely one-sided, and tend to occur in sources with stronger cores. If the relative strength of the core is due to relativistic beaming, this would suggest that the observed asymmetry of the jets close to the nucleus in these low-luminosity Fanaroff-Riley class I galaxies is also due to relativistic beaming.

\section{References}

Antonucci R.R.J., Ulvestad J.S. 1988, ApJ, 330, L97

Condon J.J., 1992, ARA\&A, 30, 575

Hummel E., Jörsäter S., 1992, A\&A, 261, 85

Hummel E., Saikia D.J., 1991, A\&A, 249, 43

Kapahi V.K., Saikia D.J., 1982, JA\&A, 3, 165

Kukula M.J. et al., 1993, MNRAS, in press

Muxlow T.W.B. et al., 1993, MNRAS, in press

Pedlar A. et al., 1993, MNRAS, in press

Saikia D.J. et al., 1990, MNRAS, 245, 397 